\title{
Advances in Optical Waveguide Engineering
}

\author{
Yakov Sidorin, MEMBER SPIE \\ Photineer Technology Group \\ and \\ University of Arizona \\ Optical Sciences Center \\ Tucson, Arizona 85721 \\ E-mail: ysidorin@photineer.com \\ Giancarlo Cesare Righini, \\ FELLOW SPIE \\ Nello Carrara Institute of Applied \\ Physics (IFAC CNR) \\ 50127 Firenze, Italy \\ E-mail: g.c.Righini@ifac.cnr.it
}

Waveguide optics now represents a truly multidisciplinary field of science and engineering, which requires significant standardization to progress technologically. The depth and breadth of waveguide optics encompass not only a rather broad range of research topics, but also a diverse variety of well-rooted industrial applications. Planar waveguide amplifiers, lasers, and the pursuit to obtain various material bases in order to realize switches, modulators, and wavelength converters are but a few examples. As the technology of waveguide optic devices and systems has matured, it has penetrated a number of markets by providing performance-competitive integrated optic and fiber-optic device solutions. This growth, in turn, requires new developments in modeling, further advances in material sciences, and innovations in integration platforms. In addition, the processing and fabrication of these new devices must be optimized in conjunction with the development of accurate and precise characterization and new testing techniques.

To address some of the advanced aspects of optical waveguide engineering, this special section comprises a variety of presentations from this amazingly interesting field. The 10 papers of the special section can be broadly grouped into the following categories: developments in active waveguide devices in rare-earth doped materials and corresponding material characterization, waveguide technology in silicon-based materials and polymers, and device design and novel experimental methodology.

Four papers on rare-earth doped waveguiding devices range from the matrix of distributed feedback lasers in active glass substrates (Bastard et al.), to investigation of already conventional (such as IOG-10) and novel glass materials (such as tungsten tellurite and zinc tellurite) as a basis for integrated optic devices (Choo et al., Nunzi Conti et al.), to realization of an original multimode pump phosphate fiber amplifier (Jiang et al.).
The following invited paper by M. R. Poulsen et al. presents a detailed overview of achievements and prospects of silicon-based waveguide technology. This ranges from the production methods for planar waveguides, including a novel method based on the utilization of focused UV-laser beams for direct waveguide imprinting, to the functionalities that are embedded into the glass materials and waveguide circuitry, to micro-ring resonators and two-dimensional photonic bandgap structures. Planar waveguide amplifiers, lasers, and the pursuit to obtain highly non-linear materials in order to realize purely glass-based switches, modulators, and wavelength converters are also presented. This overview is complimented by the paper of Cantore, proposing a novel integrated optic switch in hydrogenated amorphous silicon and crystalline silicon material base. Shacklette et al. present a comprehensive and elegant study of thermo-optic switches fabricated from very low loss newly developed acrylate polymers. The best of discussed materials exhibit losses at $1550 \mathrm{~nm}$ less than $0.1 \mathrm{~dB} / \mathrm{cm}$, which is rather exceptional for a single-mode polymer waveguide.

Finally, the novel design device and experimental methods are presented by Ghibaudo et al., discussing the wide-band integrated optic multiplexer, and by Shu, who has proposed a composite structure design of a WDM filter. The special session is concluded with the discussion of an efficient method (Aalto et al.) of mutual alignment between polarization maintaining fibers and waveguides, that would facilitate waveguide device characterization and possibly improve the efficiency of photonic integration techniques.

Interest in the development of waveguiding technologies continues to grow, which is emphasized by the addition of a bi-annual conference on waveguide fabrication to the schedule of SPIE's annual meeting and by the inclusion of an integrated optics and optoelectronics confer- 
ence in the Photonics Europe symposium, scheduled for April 2004 (the first one was held in 2002). The SPIE conference on integrated optics during the Photonics West symposium has been successfully attracting participants for seven consecutive years.

Based on the broad interest from potential contributors who were unable to complete papers in time for this issue, we believe this special section can be repeated in the future. We thank all the contributing authors and the reviewers for their timely help with selected papers, as well as the Optical Engineering staff, in particular managing editor Karolyn Labes, editorial assistant Monica Crabtree, and editor Donald O'Shea for their patient guidance and support.

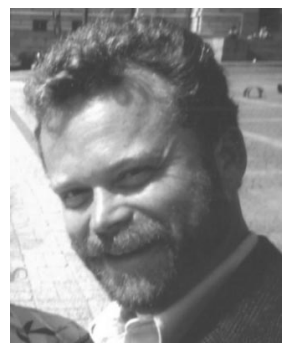

Yakov Sidorin holds a doctorate degree in optical sciences from the University of Arizona. He is a founder and a principal scientist with Photineer Technology Group and an adjunct research faculty with the Optical Sciences Center, the University of Arizona. Prior to that he was with Agilent Laboratories. His multidisciplinary experience includes integrated optics, tunable laser sources, and optical measurement techniques. As an active member of SPIE for many years, he has been organizing various conferences on integrated optics and photonics. He serves as a regular technical reviewer for journals of SPIE and OSA. This is his first editorial experience.

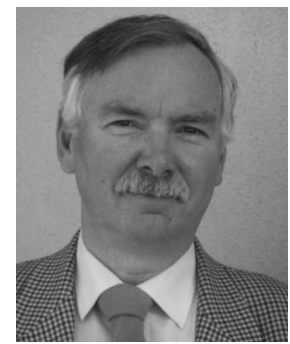

Giancarlo Cesare Righini received the "laurea" (doctor in physics) from the University of Firenze in 1967. After having been research fellow at the Institute of Physics from 1968 to 1971, since 1972 he has been on the staff of the National Research Council of Italy (CNR). Currently he is a CNR research director at the "Nello Carrara" Institute of Applied Physics (formerly IROE-CNR), head of the Optoelectronics and Photonics Dept., and responsible of the Laboratory for Optoelectronic Technologies (LAB-TOP). From 1983 to 1996 he has been director of the National Group on Quantum Electronics and Plasma Physics (GNEQP-CNR). His present research interests concern active integrated optics in glasses and other non-semiconductor materials for optoelectronics, grating structures, and nonlinear optics. He has been responsible for several international cooperation research projects. He has published over 200 papers (half of them in peer-reviewed journals), and is co-author of 6 international patents. He has presented several invited papers at international conferences. He has authored (or co-authored) various chapters in books on optoelectronics and guided-wave optics. A SPIE fellow, he has been a chairman or co-chairman of many national and international conferences, and has edited several proceedings volumes. He is member of the editorial board of various international journals, and holds positions on many boards and committees. 